\title{
Imaging of Thermal Domains in ultrathin NbN films for Hot Electron Bolometers
}

\author{
D. Doenitz, R. Kleiner, D. Koelle \\ Universität Tübingen, Physikalisches Institut - Experimentalphysik II, \\ Auf der Morgenstelle 14, D-72076 Tübingen, Germany \\ T. Scherer, K. F. Schuster \\ Institute de Radioastronomie Millimetrique, IRAM, \\ 300 Rue de la Piscine, 38406 St Martin d'Hères, France
}

(Dated: May 26, 2007)

\begin{abstract}
We present low-temperature scanning electron microscopy (LTSEM) investigations of superconducting microbridges made from ultrathin NbN films as used for hot electron bolometers. LTSEM probes the thermal structure within the microbridges under various dc current bias conditions, either via electron-beam-induced generation of an unstable hotspot, or via the beam-induced growth of a stable hotspot. Such measurements reveal inhomogeneities on a micron scale, which may be due to spatial variations in the $\mathrm{NbN}$ film or film-interface properties. Comparison with model calculations for the stable hotspot regime confirm the basic features of common hot spot models.

PACS numbers: 85.25.Am, 85.25.Pb, 07.57.Kp, 74.25.Fy
\end{abstract}

Ultrathin NbN films have recently gained much attention for use in $\mathrm{THz}$ hot electron bolometer (HEB) mixers

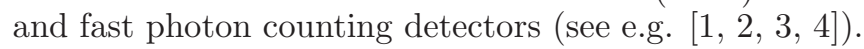
$\mathrm{NbN}$ HEB mixers are short microbridges (typical length and width of order $1 \mu \mathrm{m}$ and a few nm thickness) with contacts to antenna and dc leads. The dc current-voltage curve (IVC) is characteristic for microbridges with a supercurrent up to a critical current $I_{c}$ and a sudden jump to a hysteretic resistive behavior. Depending on film thickness, quality and geometry, typical resistances for this branch are around 100-1000 $\Omega$. When current biased on the resistive branch, the bridge stays resistive below $I_{c}$, down to a distinct voltage from where the device jumps back to the zero-voltage state at the return current $I_{r}$. When the bridge is biased at the lower stable voltage carrying region it serves as a sensitive power detector for far-infrared (FIR) radiation. The basic principle of heterodyne mixing with such a device is the beating of an incoming signal with an auxiliary local oscillator (LO) signal, producing a signal at the intermediate frequency (IF), the difference between the frequency of the input signal and the LO frequency. The resulting IF signal lays in the $0-4 \mathrm{GHz}$ range and can therefore be amplified with commercial semiconductor low-noise amplifiers. We note that for sufficient LO power, the IVC becomes non-hysteretic, and the HEB mixer is typically voltage biased.

NbN microbridge HEB mixers offer several very important advantages over other mixer devices in the $\mathrm{THz}$ range. Their small size allows to pump the mixer with very little LO power; this is an important advantage, as tunable LO sources above $500 \mathrm{GHz}$ have usually very little power output. Another advantage is due to the purely resistive nature of the device, which makes highfrequency impedance matching very easy. Because the detection is based on heating, the detection principle is not limited in frequency by specific superconducting properties, such as the energy gap of $\mathrm{NbN}$, as in the case of SIS mixers. For frequencies between 1.2 and $10 \mathrm{THz}, \mathrm{HEB}$ mixers are therefore offering currently the lowest noise. However, current NbN HEB mixers suffer from a relatively low IF bandwidth. This is due to a limited detection speed, which is often found to be below $2 \mathrm{GHz}$. For $\mathrm{THz}$ applications, IF bandwidths up to $10 \mathrm{GHz}$ are however desirable in radio astronomy. The detailed physics of the involved time constants, which ultimately limit the IF bandwidth, is therefore a subject of ongoing research in many different laboratories.

While first results for these applications are promising [5, [6], modelling of the devices is quite complex. As a consequence, the impact of fabrication and specific material parameters on device performance remains unclear, and optimization is slow and based on trial and error. Beyond first lumped element models 7], more elaborated models have been proposed [8, 9], which describe the physics of the microbridges in a spatially resolved manner. Most of the numerical and analytical spatially distributed models are based on assumptions first made for superconducting bridges by Skocpol, Beasley and Tinkham [10. In this approach the resistive behavior of a superconducting microbridge is modelled through the thermal equilibrium between a normal conducting and therefore dissipating hot spot and the substrate. The lateral thermal conduction and the cooling to the substrate determine the detailed shape of the hot spot. The general solution within this analytical frame work is a symmetrical hot spot geometry centered between the contacts. Although some convincing results, such as modelling of rf-pumped IVCs and bias dependent conversion gain curves could be obtained for the current NbN HEBs within the existing distributed models, the existence of a single centered hot spot geometry for these devices has not been directly shown so far.

Over the last two decades, low-temperature scanning electron microscopy (LTSEM) has been used to provide local information (on a micron scale) on various properties of superconducting thin films and Josephson junctions, such as the spatial distribution of the transition temperature $T_{c}$ and critical current density $j_{c}$, or on Josephson vortices in long junctions 11, 12, 13], and on Abrikosov vortices [14, 15] and supercurrent distribution [16] in SQUID washers. Very early, 

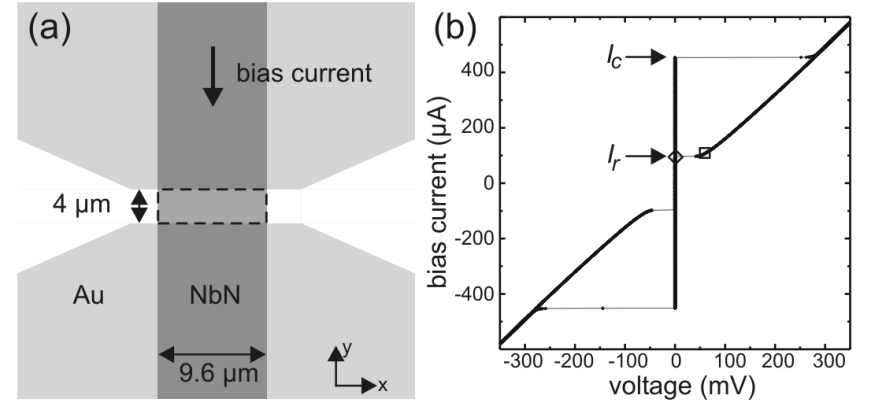

FIG. 1: (a) NbN HEB layout: $W=9.6 \mu \mathrm{m}$ wide NbN strip, partially covered by Au pads with $L=4 \mu \mathrm{m}$ separation. (b) IV characteristic measured at $T \approx 5 \mathrm{~K}$ in the LTSEM. Open diamond and circle show bias points for LTSEM images shown in Fig. 2(b) and Fig. 3, respectively

LTSEM has been applied to proof the concept of hot spot formation in long and thick superconducting bridges [17, 18, 19]. In this paper we show that LTSEM can also be applied to investigate the thermal structures of microbridges from ultrathin $\mathrm{NbN}$ films with much smaller geometries, approaching those of HEBs.

The samples which we investigated were fabricated as follows: Thin $\mathrm{NbN}$ films were deposited from a 4 inch $\mathrm{Nb}$ target by $13.56 \mathrm{MHz}(240 \mathrm{~W})$ rf magnetron sputtering on a 2 inch fused quartz substrate at room temperature in a $0.852 \mathrm{~Pa} \mathrm{~N} / 2 / \mathrm{Ar} / \mathrm{CH}_{4}$ atmosphere (gas flow: 2.7, 46 and $0.6 \mathrm{sccm}$, respectively). This process yields typically $T_{c}=11 \mathrm{~K}$ for $t=5 \mathrm{~nm}$ thick films and $T_{c}=15 \mathrm{~K}$ for $t>20 \mathrm{~nm}$. The $\mathrm{NbN}$ films were patterned by reactive ion etching in a $\mathrm{CF}_{4} / \mathrm{O}_{2}$ mixture to form long microbridges of width $W$. Subsequently, $50 \mathrm{~nm}$ thick Au contact pads were formed on top of the $\mathrm{NbN}$ bridges, with a separation, which defines the length $L$ of the active region of the NbN HEB, as shown in Fig. 1(a). Typical device geometries vary from $L \times W=0.5 \mu \mathrm{m} \times 4 \mu \mathrm{m}$ to $5 \mu \mathrm{m} \times 10 \mu \mathrm{m}$. We present results from a device with $t=5 \mathrm{~nm}, L=4 \mu \mathrm{m}$ and $W=9.6 \mu \mathrm{m}$, which shows a clearly hysteretic IVC at $T \approx 5 \mathrm{~K}$ [c.f. Fig. 1(b)]. We note that all data presented here have been obtained with current bias.

For imaging by LTSEM, the sample was mounted on a liquid He cooled stage and operated at a temperature $T \approx 5 \mathrm{~K}$. The local perturbation by the focused electron beam (e-beam) induces an increase in temperature $\delta T(x, y)$ on the sample surface in the $(x, y)$ plane, on a length scale of approximately $1 \mu \mathrm{m}$, which determines the spatial resolution of this imaging technique, and with a maximum local increase in temperature $\Delta T\left(x_{0}, y_{0}\right)$ of a fraction of $1 \mathrm{~K}$, centered on the beam spot position $\left(x_{0}, y_{0}\right)$ on the sample surface [11]. For modelling the local perturbation we have used a combination of Monte-Carlo calculations for the beam energy deposition and finite element modelling (FEM) of the corresponding thermal plume. However, simplified semianalytical models turned out to give very similar results [13]. The local change in $T$ may change global properties of the bridge, e.g. the voltage $V$ across the current-biased bridge. This voltage change $\delta V$ depends on the e-beam position $\left(x_{0}, y_{0}\right)$ and thus can be recorded to obtain a $\delta V\left(x_{0}, y_{0}\right)$-image. To improve the signal-to-noise ratio, we use a beam-blanking unit operating at $5 \mathrm{kHz}$ and detect $\delta V$ with a lock-in amplifier.

In order to create a voltage drop along the microbridge, a continuous domain (hot spot) of normal conducting film across the bridge is required. Such a domain can be generated either by resistive heating due to the bias current $I_{b}>I_{r}$ or, for lower bias currents, by the combined effect of e-beam and current heating. It is therefore possible to distinguish two different experiments:

(A) e-beam-induced generation of (unstable) hot spot: If biased below $I_{r}$, the device may switch to a resistive state upon e-beam irradiation due to generation of an unstable hotspot (i.e. the hotspot disappears when the e-beam is turned off). The lock-in detected voltage signal corresponds to the voltage induced by the (unstable) hotspot. (B) e-beam induced growth of (stable) hotspot: If biased slightly above $I_{r}$ in the resistive state, a stable hotspot is generated, which size may be altered upon e-beam irradiation. The lock-in detected voltage signal corresponds to the beam spot position-dependent small change in voltage drop due to the beam-induced extension of the otherwise self-sustained hotspot.

We first discuss imaging mode (A). Figure 2(a) shows IVCs recorded during e-beam irradiation (for various values of the beam current $I_{e l}$ and fixed beam voltage $U_{e l}=20 \mathrm{kV}$ ) at a fixed position on the center of the device, and for comparison, the IVC without irradiation. With increasing $I_{e l}$ the critical current is reduced, while the normal resistance remains almost unchanged. Under sufficiently strong e-beam irradiation $\left(I_{e l} \gtrsim 0.2 \mathrm{nA}\right)$ the hysteresis in the IVC vanishes. The general shape of the IVCs with e-beam irradiation is surprisingly similar to IVCs of rf pumped devices [8]. This is a strong indication that a general mechanism, which is likely to be of thermal nature, describes the physics of the IVCs for very different types of energy input.

Figure 2(b-d) shows LTSEM voltage images recorded at slightly sub-critical current bias $\left(I_{b} \lesssim I_{r}\right)$ for fixed $U_{b}$ and different values of $I_{e l}$. These images reflect the lo-

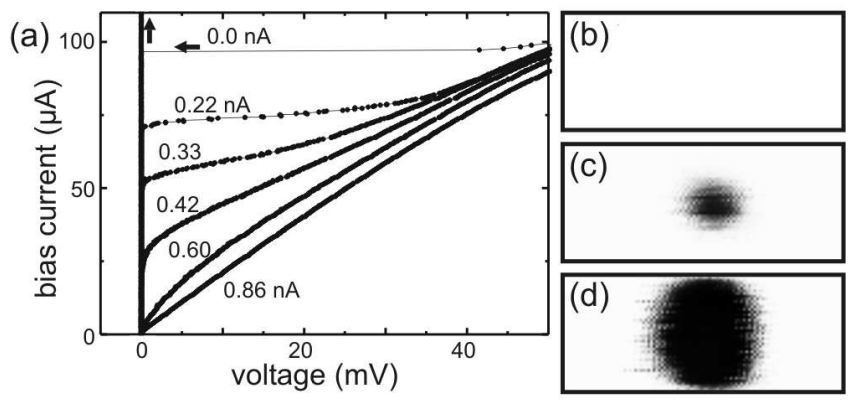

FIG. 2: (a) Non-hysteretic IVCs for e-beam irradiation on the center of the device $\left(I_{e l}\right.$ from 0.22 to $\left.0.86 \mathrm{nA} ; U_{e l}=20 \mathrm{kV}\right)$; unirradiated hysteretic IVC is shown for comparison (arrows indicate sweep direction of $I_{b}$ ). (b-d) LTSEM voltage images $\left(I_{b} \approx 95 \mu \mathrm{A}\right.$, i.e. just below $I_{r}, U_{e l}=10 \mathrm{kV}$ ) for different $I_{e l}$ : (b) $0.24 \mathrm{nA}$, (c) $0.26 \mathrm{nA}$, (d) $0.28 \mathrm{nA}$. The rectangles mark the boundaries of the sample [c.f. dashed box in Fig[1(a)]. 


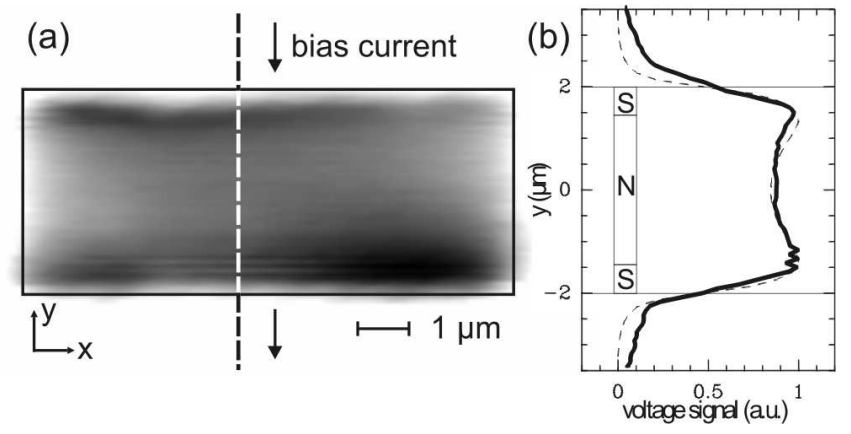

FIG. 3: (a) LTSEM voltage image at $I_{b}=110 \mu \mathrm{A}$ (stable hot spot condition); $I_{e l}=10 \mathrm{pA}, U_{e l}=5 \mathrm{kV}$. Dark areas indicate high voltage responsivity to e-beam irradiation. (b) Linescan of LTSEM voltage signal (solid curve) along the dashed line in (a) and comparison with model calculation using 2dimensional FEM techniques (dashed curve). Inset indicates the transition between superconducting $(\mathrm{S})$ and normal $(\mathrm{N})$ region at $y= \pm 1.4 \mu \mathrm{m}$.

cal sensitivity to e-beam irradiation for triggering of an unstable hot spot. For $I_{e l}=0.24 \mathrm{nA}(\mathrm{b})$, no hot spot is induced at all. With an increase to $I_{e l}=0.26 \mathrm{nA}$ (c) a small spot on the image appears: only when the ebeam is applied within this spot a voltage is induced. As expected from thermal and electrical symmetry considerations, the spot is centered with respect to the $x$-axis. It is also centered with respect to the $y$-axis (along the bridge), which can be easily explained by the additional cooling effect provided by the gold pads. With further increase of $I_{e l}$ to $0.28 \mathrm{nA}$, the spot increases rapidly [see Fig. 2(d)]. An analysis of the shape of the spots leads to the estimate of the spatial resolution of $\approx 0.4 \mu \mathrm{m}$ for this type of measurement.

For imaging mode $(\mathrm{B}),\left(I_{b}>I_{r}\right)$, i.e. in the area of a stable bias current driven hot spot, the voltage across the sample increases slightly under irradiation due to the following effect: The beam-induced increase in temperature causes the hot spot to grow; thus an increased region of the $\mathrm{NbN}$ film becomes resistive, and the normal resistance $R$ is increased. This mechanism is most effective for an e-beam position close to the superconducting/normal conducting $(\mathrm{S} / \mathrm{N})$ boundary. The result is a characteristic double ridge picture, with the maximum of the signal indicating the location of these lateral bound- aries, as shown in Fig. 33(a). However, the LTSEM signal also reveals a clear variation along the $\mathrm{S} / \mathrm{N}$ boundaries. This may be due to inhomogeneities in the film quality such as thickness or composition, or due to local variations in the thermal coupling to the substrate or contact pads. The detected inhomogeneities will result in an asymmetric current density and may adversely affect device performance.

We modelled the device under the stable hotspot bias conditions as in imaging mode (B) by a 2-dimensional finite element calculation, taking into account the heat transfer to the substrate as balanced by the heat production by the bias current dissipation. The e-beam induced change of the substrate surface temperature was modelled using Monte-Carlo techniques to compute the energy input and a finite element model in the symmetry plane of the e-beam axis to derive the temperature resulting from this energy input. For the parameters $\left(I_{b}, I_{e l}, U_{e l}\right)$ and device geometry used in the experiment [c.f. Fig 3, the model predicts a superconducting $(\mathrm{S}) /$ normal conducting $(\mathrm{N})$ transition at a position of $y= \pm 1.4 \mu \mathrm{m}$, as indicated in Fig 3(b). The derived voltage signal $\delta V(y)$ for a linescan along the $y$-direction [c.f. Fig. 3(b)] reproduces nicely the characteristic double peaked shape as observed experimentally, and matches also very well the general form of the measured curve.

In conclusion, we demonstrated that LTSEM is a useful tool to investigate thermal domain (hotspot) formation in ultrathin superconducting films. Our experiments show that for current bias below $I_{r}$, e-beam irradiation induces a hotspot, depending on deposited beam energy, beam spot position and sample inhomogeneity. For biasing above $I_{r}$, i. e. in the regime of a stable hotspot, comparison of measurements with 2-dimensional modelling shows that the classical hotspot model does apply. Our investigations also show that inhomogeneities on a micron scale can be detected. Further investigations are required to clarify the nature of such inhomogeneities and their impact on device performance. Furthermore, it will be interesting to include RF pumping to investigate the hotspot formation under typical working conditions of superconducting hot electron bolometers.

D.D. gratefully acknowledges support from the Evangelisches Studienwerk e.V. Villigst. This work was supported by the EU FP6 Program RADIONET (AMSTAR).
[1] Y. P. Gousev et al., J. Appl. Phys. 75, 3695 (1994).

[2] J. Kawamura et al., J. Appl. Phys. 80, 4232 (1996).

[3] T. Lehnert et al., J. Appl. Phys. 83, 3892 (1998).

[4] G. N. Gol'tsman et al., Appl. Phys. Lett. 79, 705 (2001).

[5] S. Cherednichenko et al., Physica C 372-376, 427 (2002).

[6] M. C. Wiedner et al., A\&A 454, L33 (2006).

[7] B. S. Karasik, A. I. Elantiev, Appl. Phys. Lett. 68, 853 (1996).

[8] D. W. Floet et al., Appl. Phys. Lett. 74, 433 (1999).

[9] H. F. Merkel et al., IEEE Trans. MTT 48, 690 (2000).

[10] W. J. Skocpol et al., J. Appl. Phys. 45, 4054 (1974).
[11] J. R. Clem, R. P. Huebener, J. Appl. Phys. 51, 2764 (1980).

[12] R. P. Huebener, Rep. Prog. Phys. 47, 175 (1984).

[13] R. Gross, D. Koelle, Rep. Prog. Phys. 57, 651 (1994).

[14] D. Doenitz et al., Appl. Phys. Lett. 85, 5938 (2004).

[15] R. Straub et al., Appl. Phys. Lett. 78, 3645 (2001).

[16] D. Doenitz et al., Phys. Rev. B 73, 064508 (2006).

[17] R. Eichele et al., Appl. Phys. Lett. 38, 383 (1981).

[18] R. Eichele et al., Z. Phys. B 48, 89 (1982).

[19] R. Eichele et al., J. Low. Temp. Phys. 52, 449 (1983). 